\title{
Identifying the Intended Addressee in Mixed Human- Human and Human-Computer Interaction from Non-verbal Features
}

\author{
Koen van Turnhout ${ }^{*}$, Jacques Terken*, Ilse Bakx ${ }^{\dagger}$, Berry Eggen* \\ * Dept. of Industrial Design \\ Eindhoven University of Technology, The Netherlands

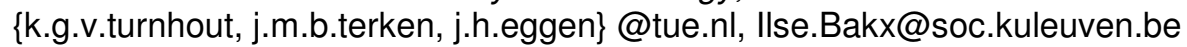

\begin{abstract}
Against the background of developments in the area of speechbased and multimodal interfaces, we present research on determining the addressee of an utterance in the context of mixed human-human and multimodal human-computer interaction. Working with data that are taken from realistic scenarios, we explore several features with respect to their relevance to the question who is the addressee of an utterance: eye gaze both of speaker and listener, dialogue history and utterance length. With respect to eye gaze, we inspect the detailed timing of shifts in eye gaze between different communication partners (human or computer). We show that these features result in an improved classification of utterances in terms of addressee-hood relative to a simple classification algorithm that assumes that "the addressee is where the eye is", and compare our results to alternative approaches.
\end{abstract}

\section{Categories and Subject Descriptors}

H.5.3 [Information Interfaces]: Multimedia Information Systems - User Interfaces voice I/O. J.4 [Social and Behavioral Sciences]: Psychology

\section{General Terms \\ Human Factors; Algorithms}

\section{Keywords}

Perceptive user interfaces, multi party interaction, eye gaze.

\section{INTRODUCTION}

Most research on the application of speech in the interface implicitly assumes a 'single user - single system' scenario. However, many applications involving speech in fact allude to situations where the presence of multiple people is highly likely and interaction with the application is interleaved with interaction between humans. At the most basic level, someone dictating a letter in his office may be distracted by a question from the secretary that requires a reply. At a more sophisticated level, we may think of people interacting with a speech based database

Permission to make digital or hard copies of all or part of this work for personal or classroom use is granted without fee provided that copies are not made or distributed for profit or commercial advantage and that copies bear this notice and the full citation on the first page. To copy otherwise, or republish, to post on servers or to redistribute to lists, requires prior specific permission and/or a fee.

Conference'04, Month 1-2, 2004, City, State, Country.

Copyright 2004 ACM 1-58113-000-0/00/0004 ..\$5.00 query system such as an integrated trip planner or an automated real estate agent, where the interaction with the system is interleaved with discussions between the users about search parameters or intermediary results.

Current generation systems, lacking contextual awareness, will assume that all the speech that is registered at the microphone is intended for the system, and will in many cases reply with "I'm sorry, I didn't understand, could you please repeat". This is not only incorrect, as it reflects that the system wrongly assumes to be the addressee of all utterances, it is also socially inappropriate since it interrupts (and likely disrupts) the human-human conversation. In order to deal with this problem, we need to build systems that are aware of the communicative context so that they understand who is being addressed and may display socially appropriate behaviour. Besides improving the communicative capabilities of the system, context awareness may also increase system performance by filtering out speech that is not intended to be recognized by the system. The aim of the current paper is to explore behavioral features that may help to decide who is the addressee of an utterance in a context of mixed human-human and human-system interaction.

\subsection{Modality cues to addressee-hood}

Cues that help to decide on the addressee of an utterance may be both verbal and non-verbal. With respect to verbal cues, in the context of a meeting the speaker may address another person explicitly by addressing him/her by name. With respect to system design, early systems incorporated this observation by requiring the user to explicitly address the computer ("Computer, ...") or to press a push-to-talk button to make clear that the speaker is about to address the system. However, usability studies point out that this is an awkward solution, as people forget to address the system or press the push-to-talk button consistently. This motivates the search for a more advanced solution.

In the context of system design, a more technical solution exploiting the verbal modality has been explored by Katzenmaier et al. [3], who investigated whether the degree to which an incoming utterance fits the language model informs about addressee-hood. This approach was inspired by the observation that speech-centric multimodal interfaces have much more restricted language models than humans. However, it is also clear that this strategy has its limitations, as many utterances that perfectly fit the language model could equally well be intended for the system or for someone else (e.g. confirmations such as "yes").

Building on work in conversation analysis and social psychology, alternative approaches have been explored exploiting non-verbal 
cues, primary those related to eye gaze. As has been shown already in the sixties and seventies, eye gaze serves an important role in guiding the conversation, both at the side of the speaker and the listener (e.g. [4],[6]). At the speaker's side, looking at the listener may serve the function of monitoring the attention level and the processing status of the incoming speech, and help to regulate the flow of conversation. At the listener's side, looking at the speaker serves both the function of providing feedback for the speaker's monitoring activity, to inspect the speaker's behaviour (facial expression, posture etc) for information about the speaker's attitude and emotion, and to monitor for nonverbal cues for turntaking. Most of these early findings concerning the use of nonverbal cues in communication are based on the analysis of dyadic conversations. Later research on triadic and multiparty conversations has confirmed and extended the early findings. Vertegaal et al. [10] provide evidence that gaze behaviour is a reliable predictor of addressee-hood. Likewise, Takemae et al. [9] provide evidence that gaze behaviour of the speaker is an indication of addressee-hood and has a regulatory function in turn-management (although their evidence is more anecdotal in nature). Thus, it is concluded that the speaker's eye gaze provides information about whom the speaker is addressing.

In the context of mixed human-human and human-computer interaction yet another factor comes into play. A database information query system with a speech-centric multimodal user interface cannot be simply equated with a third party in humanhuman interaction. A study on mixed human-human and humancomputer interaction by Bakx et al. [2] found that the trends for eye gaze behaviour that had been found in multiparty humanhuman conversations did not apply. Even when the human participants were addressing each other, most of the time they looked at the computer screen. This behaviour could be well explained by Argyle's [1] situational attractor hypothesis, which states that objects that play a central role in a task that the participants in human-human conversation are performing, attract the visual attention, thereby overruling trends for eye gaze behaviour in human-human conversation as summarized above. Thus, the situation of mixed human-human and human-computer interaction is more complicated, and human eye gaze behaviour in such situations cannot directly be used as a cue to addressee-hood. In the current paper, we further explore visual attention patterns in mixed human-human and human-computer interaction.

The specifc character of human-human and human-computer interaction also opens up specific opportunities for detecting the addressee of utterances. As has been pointed out in [2] and [3] users tend to speak differently to systems, and this can be detected by low level linguistic features, such as utterance length and number of words. Truly interactive situations like the one described in this paper also enable to take system events into account. The communicative repertoire of systems is limited compared to that of humans and as such system actions may structure the dialog and evoke very specific reactions from users. For example, after a question from the system users will often respond with an answer.

\subsection{Methodological note}

Algorithms determining the addressee of an utterance in situations of mixed human-human and human-computer interaction have been proposed in other publications as well (e.g. [7],[5]). However, the focus of these papers is more on developing computer vision algorithms that deal with head or body orientation than with collecting data on human behavior in realistic situations. As a result, simplifying assumptions are made about the way humans behave in such situations, which suffice to demonstrate the robustness of the computer vision algorithms but are inadequate when they have to deal with human behavior in realistic situations. On the other hand, the research by Katzenmaier et al. [3] and the research project from which the current paper and the earlier paper [2] emerge are precisely intended to develop algorithms that are able to deal with realistic behaviour.

\subsection{Paper outline}

The main focus of this paper is the exploration of behavioral cues that could potentially be used for classification. We discuss facial orientation, utterance length and reactions on system events. In order to evaluate the potential integration of these features, we present a straightforward Naïve Bayes classifier. We convert each behavioral type into a set of between 4 and 8 mutually exclusive classes. Based on these 3 feature vectors, we make a combined decision and evaluate this with ROC curves. A comparison with an 'the addressee is where the eye is' classifier is given and questions of generality are discussed. In section 2 we describe our experimental setup and data collection. Section 3 elaborates the behaviour of the participants and the definition of features. Section 4 describes the classifier, evaluated in Section 5. In Section 6 conclusions are given and future work is briefly discussed.

\section{EXPERIMENTAL SET-UP AND DATA COLLECTION}

A Wizard of Oz set-up was created in order to collect data. This was necessary as the current operational system (see[8]) has no contextual awareness and assumes all incoming speech is intended for the system. The functionality of the wizard set-up closely mimicked the functionality of the system described in [8]. It enables users to obtain information about train departure and arrival times in the Netherlands. The system includes an information kiosk displaying a form containing the search parameters for the system: departure station, arrival station, date of travel, time of travel, and a parameter indicating whether the time of travel is the desired departure or arrival time. The system supports both mixed-initiative spoken dialogue and tap-and-talk: users can enter values for the different search parameters by means of speech, both as a reply to a system request for information and by tapping on a field of the form on the touch screen of the information kiosk. The system responds to the input by filling the appropriate field in the form with text, and by prompting for the next empty field to be filled by means of synthesized speech.

The speech processing and control modules of the original system were replaced by a wizard operating a GUI interface, allowing him to provide the data from the database and to control the system prompts. Speech recognition errors and corresponding system behavior [8] where faked but no formal protocol was followed to control the amount of speech recognition errors. The system produced utterances only when it was appropriate to do so in the communicative setting: the system prompt for filling the next empty field was produced immediately after the previous field was processed, unless the participants were already involved 
in further discussion: in that case the prompt was withheld. Users were not required to answer system prompts immediately; in addition they could provide query parameters in any order. The decision to accept an utterance as intended for the system, whether or not explicitly indicated by users as such, was up to the wizard.

Eleven pairs of people, of mixed age, sex and educational background, who were already acquainted with each other were invited to the usability lab of the university. They were briefly explained how the information system worked and had the opportunity to practice. Next they were introduced to the experimental task. The task consisted of planning two roundtrips, to tourist attractions in the Netherlands, one to a museum and one to a zoo. They received information about the tourist attractions and constraints on the desired outcomes (for instance "you want to return home at a reasonable time"). In order to stimulate discussion and negotiation during the conversation with the system, participants forming a pair received different information about the tourist attractions (recommending different sites) and different constraints (although not conflicting). Participants were asked to conduct the negotiation during the interaction with the system instead of having the negotiations prior to the interaction.

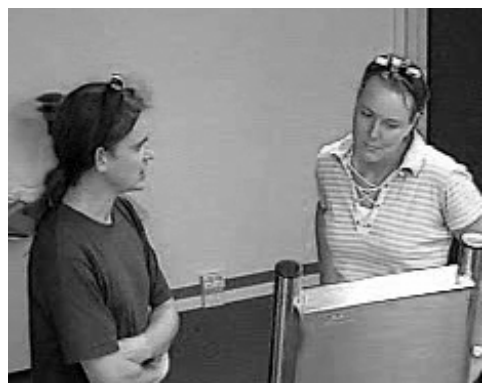

Figure 1: Video frame showing two participants interacting with the information kiosk.

Due to technical problems, data for three pairs had to be dismissed, so that 32 dialogues remained for further analysis (eight pairs, four dialogues per pair). Transcripts of all dialogues were made by hand, and video recordings were inspected to annotate eye gaze for both participants manually. ${ }^{1}$ Intuitively, participants took positions so that they formed a triangle with the information kiosk, with corners of about $60^{\circ}$ (see Figure 1). As a result, shifting eye gaze between screen and the other participant required a shift in head orientation. Eye gaze was classified either as "looking at the screen" or "looking at partner" for each video frame. When looking away from the partner, participants might in principle look elsewhere than at the screen, but if they did so at least their head was turned into the direction of the screen so that "looking elsewhere" was not transcribed as a separate category.

The audio recording was segmented into utterances. An utterance was defined as a stretch of speech from the same speaker, preceded and followed by silence for at least $500 \mathrm{msec}$. This

${ }^{1}$ During the sessions, also measurements were made of the head orientations of the participants by means of infrared tracking with specially developed devices. The head tracking data will not be discussed in this paper, but measurements showed a high agreement between manual annotations of eye gaze and head tracking data. criterion was determined empirically, and was found to give a good match with the segmentation into utterances on the basis of the content. In all, the 32 dialogues contained 925 utterances, 202 directed to the system and 723 directed to the other member of the pair, suggesting that our instruction for people to negotiate during the interaction with the system had been quite successful; it should be noted, though, that we do not claim that this is the normal situation: in other communicative contexts the distribution between utterances for the system (or a human travel or real estate agent) and for the partner may be quite different, depending on the need for negotiation.

\section{FEATURES}

In this section we analyze the behavior of participants during utterances for the system and utterances for the partner in order to find potential features.

\subsection{Eye-Gaze}

The behavior of the speaker and listener were coded into 4 states. Table 1 shows the possible states, for further reference they are labeled A to D.

Table 1: Coding of possible states.

\begin{tabular}{|l|l|}
\hline Event & Score \\
\hline Both participants look at the system & A \\
\hline $\begin{array}{l}\text { The speaker looks at the system but the partner looks } \\
\text { at the speaker }\end{array}$ & B \\
\hline $\begin{array}{l}\text { The speaker looks at the partner but the partner looks } \\
\text { at the system }\end{array}$ & C \\
\hline Both participants look at each other & D \\
\hline
\end{tabular}

The order of states is not chosen randomly: the states are ordered from $\mathrm{A}$, highest visual attention to the system to $\mathrm{D}$, lowest visual attention to the system. State $\mathrm{C}$ is considered lower in attention to the system than state $\mathrm{B}$, because we put more weight on the behaviour of the speaker.

There may be one or more transitions between states during an utterance. In Table 2 the relative distributions of utterances with and without changing states are listed.

Table 2: Occurrence of state transitions during utterances for the system and for the partner

\begin{tabular}{|l|c|c|}
\hline \multicolumn{1}{|c|}{ In utterances for: } & System & Partner \\
\hline Transitions do not occur & $144(71 \%)$ & $404(56 \%)$ \\
\hline Transitions occur & $58(29 \%)$ & $319(44 \%)$ \\
\hline & 202 & 723 \\
\hline
\end{tabular}

In the following two sections we will examine utterances without transitions and utterances with transition separately.

\subsubsection{Utterances without transition}

In this section we will se whether the observed state is informative for the addressee of the utterance Table 3: shows the relative occurrence of utterances for the system and partner for each state. 
The "addressee is where the eye is" rule predicts the behavior in case of utterances for the system quite well: $95 \%$ of the utterances intended for the system is in state $\mathrm{A}$ or $\mathrm{B}$, meaning that the speaker looks at the system. However the utterances for the partner are badly predicted: only $42 \%$ of the utterances for the partner is in state $\mathrm{C}$ or $\mathrm{D}$, meaning that the speaker looks at the partner effectively close to chance. This confirms our earlier finding that the system acts as a situational attractor.

Table 3: Relative occurrence of state A-D utterances for the system and utterances for the partner without transition

\begin{tabular}{|l|c|r|}
\hline State & \multicolumn{1}{|l|}{ System } & \multicolumn{1}{|l|}{ Partner } \\
\hline A & $126(88 \%)$ & $182(45 \%)$ \\
\hline B & $10(7 \%)$ & $52(13 \%)$ \\
\hline C & $3(2 \%)$ & $44(11 \%)$ \\
\hline D & $5(3 \%)$ & $126(31 \%)$ \\
\hline & 144 & 404 \\
\hline
\end{tabular}

\subsubsection{Utterances with transitions}

Transitions in utterances can occur for multiple reasons. In utterances for the partner they are quite common. As Kendon [4] notes in dyadic conversation speakers tend to look away from their partner near the beginning of an utterance (mainly attributed to reducing cognitive input), and look at the partner near the end of an utterance (having multiple functions). In our setup looking away from the partner equates looking at the system. Given this background transitions are most likely to occur in utterances that are intended for the partner. However transitions also occur during utterances for the system. One obvious example is when the speaker turns towards the system (e.g. from a state of mutual gaze) to address the system during the utterance instead of turning to the system before the start of the utterance. Another example is the occurrence of utterances (as defined above) that consist of two parts, one for the partner and one for the system ("composites" $\mathrm{N}=21$ ).

Table 4 , listing the number of transitions found in utterances for the system and utterances for the partner, shows utterances having multiple transitions to be quite likely. In the remainder of the paper we do not make a difference between utterances with a single or more transitions.

Table 4: Occurrence of transitions during utterances for the partner expanded to number of transitions

\begin{tabular}{|l|r|r|}
\hline \multicolumn{1}{|c|}{ In utterances for: } & \multicolumn{1}{l|}{ System } & \multicolumn{1}{l|}{ Partner } \\
\hline Single Transition & $38(66 \%)$ & $185(58 \%)$ \\
\hline Two Transitions & $14(24 \%)$ & $85(27 \%)$ \\
\hline Three Transitions & $3(5 \%)$ & $23(7 \%)$ \\
\hline Four Transitions or more & $3(5 \%)$ & $26(8 \%)$ \\
\hline & 58 & 319 \\
\hline
\end{tabular}

\subsubsection{Occurrence of states}

Since the occurrence of states was found to be a discriminating factor for utterances without transition, we also look at the frequency of occurrence of states in utterances with transitions. Table 5 shows the results. Note that the sum here does not equal the number of utterances, since an utterance can have multiple states.

Within utterances for the system state $\mathrm{A}$ is frequent and state D rare but the effect is much weaker than the effect shown in Table 3. As such further refinement is desirable.

Table 5: Number of utterances for the system and partner with a transition containing each state

\begin{tabular}{|l|r|r|}
\hline State & \multicolumn{1}{|l|}{ System } & \multicolumn{1}{l|}{ Partner } \\
\hline A & $26(39 \%)$ & $78(21 \%)$ \\
\hline B & $19(28 \%)$ & $93(24 \%)$ \\
\hline C & $16(24 \%)$ & $121(32 \%)$ \\
\hline D & $6(9 \%)$ & $88(23 \%)$ \\
\hline & 67 & 380 \\
\hline
\end{tabular}

\subsubsection{Timing of state changes}

Next we investigate whether separation can be improved if we take timing of state changes into account. Of particular relevance might be the occurrence of states at the beginning and end of the utterance. Table 6 lists the begin and end states of utterances for the system, Table 7 does the same for utterances for the partner. Some utterances end in the same state as they begin, naturally these must have at least two transitions.

Table 6: Start and end states of utterances for the system

\begin{tabular}{|l|r|r|r|r|r|}
\hline & Start A & Start B & Start C & Start D & \\
\hline End A & 5 & 17 & 7 & 7 & $61 \%$ \\
\hline End B & 5 & 4 & 1 & 2 & $20 \%$ \\
\hline End C & 8 & 0 & 0 & 0 & $14 \%$ \\
\hline End D & 0 & 2 & 0 & 0 & $3 \%$ \\
\hline & $31 \%$ & $40 \%$ & $14 \%$ & $16 \%$ & \\
\hline
\end{tabular}

Table 7: Start and end states of utterances for the partner

\begin{tabular}{|l|r|r|r|r|r|}
\hline & Start A & Start B & Start C & Start D & \\
\hline End A & 16 & 28 & 26 & 19 & $28 \%$ \\
\hline End B & 15 & 6 & 7 & 17 & $14 \%$ \\
\hline End C & 52 & 8 & 7 & 24 & $29 \%$ \\
\hline End D & 16 & 32 & 19 & 27 & $29 \%$ \\
\hline & $31 \%$ & $23 \%$ & $18 \%$ & $27 \%$ & \\
\hline
\end{tabular}

As can be seen, the begin state is not very informative. However, states at the end of the utterance show a more meaningful pattern: there appears to be a tendency for the speaker (and preferably both participants) to look at the system at the end of utterances for the system. This 'last state' effect is an improvement over looking at the mere occurrence of states.

A more detailed look at timing could possibly improve this 'last state' effect. Figure 2 shows the timing of shifts. On the horizontal axis the delay between the beginning of the utterance and the transition is plotted, on the vertical axis the distance in time between the transition and the end of the utterance. We show four plots: (a) transitions away from the system during utterances for the partner; (b) transitions towards the system during utterances 
for the partner; (c) transitions away from the system during utterances for the system; (d) and transitions toward the system during utterances for the system. Each plot is cropped to an area of $4 \mathrm{~s}$ by $4 \mathrm{~s}$.
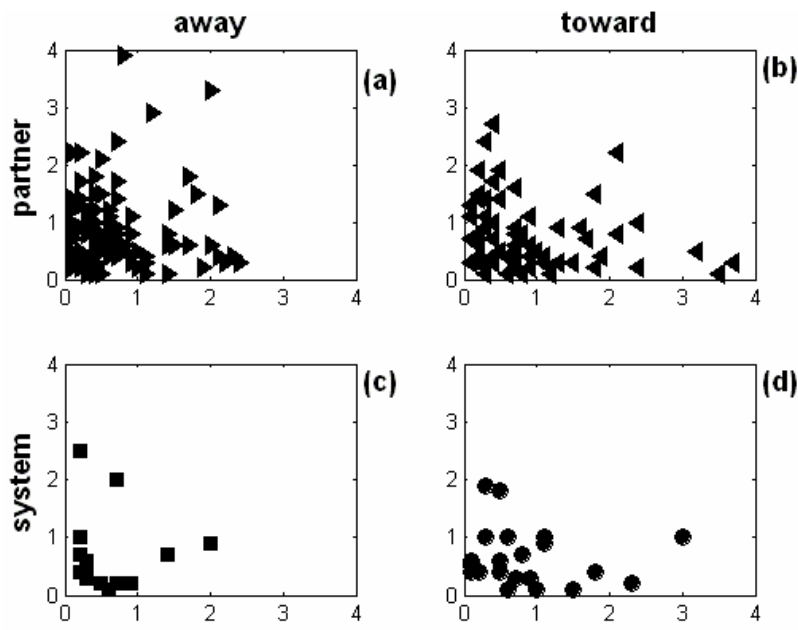

Figure 2: Timing of transitions as a function of delay since utterance onset and distance in time to utterance end: (a) transitions away from the system during utterances for the partner; (b) transitions towards the system during utterances for the partner; (c) transitions away from the system during utterances for the system; (d) transitions towards the system during utterances for the system. In each figure the horizontal axis represents the distance between the transition and the end of the utterance and the vertical axis represents the delay between utterance onset and the transition.

As can be seen, during utterances for the partner, shifts away from the system (a) are spread more along the vertical axis and shifts towards the system (b) along the horizontal axis. There appears to be a trend, then, that shifts away from the system are positioned near the end of an utterance, while shifts toward the system occur near the beginning of an utterance. This effect is consistent with the findings by Kendon [4], mentioned earlier this section. Since the number of utterances for the system in this category is rather low and there are no clear trends visible, we cannot draw conclusions about the behavior during utterances toward the system. It is however not likely that we would find a reverse effect for these utterances as such an effect would imply that people look at the partner to reduce input interfering with cognitive processing. We analyzed similar graphs containing all transition types, but no salient effects where found. Thus the best way to deal with transition timing is to look at the last state observed during the utterance.

\subsubsection{Transitions after the end of utterances}

Another question we may ask is whether taking the exact utterance boundaries for detecting 'last state' is actually best. Pairs seem to look toward the system at the end of an utterance for the system to monitor system output. This gaze and the continuation of the dialog may well be prolonged until system output arrives. If so two effects should be observed: first after utterances for the system people tend look toward the system more often then after utterances for the partner. Second after utterances for the system a longer silence can be observed than after utterances for the partner.
Figure 3 shows both effects. The lines indicate utterances that are in state A. Utterances for the system are marked with a cross and utterances for the partner with a dot. We only expect to find state A if the next utterance has not yet started. If the next utterance has started at time $t$, the utterance is excluded from analysis. The markers without line show relative numbers of these 'utterances under analyses'. Both the graphs for the system and partner are scaled towards the total number of utterances for those types.

In the graph we see that the lines indicating the relative number of utterances in state A are roughly parallel. As such measuring the end state after the utterance does not add much information. Measuring the silence after the utterance does, since the number of utterances for the partner that have not yet been followed by a follow up utterance drops quite fast after ending the utterance, where this is not the case for utterances for the system. However, using this feature would in practical implementation lead to delaying the decision whether an utterance is intended for the system or not. As such we have not implemented this in the classifier.

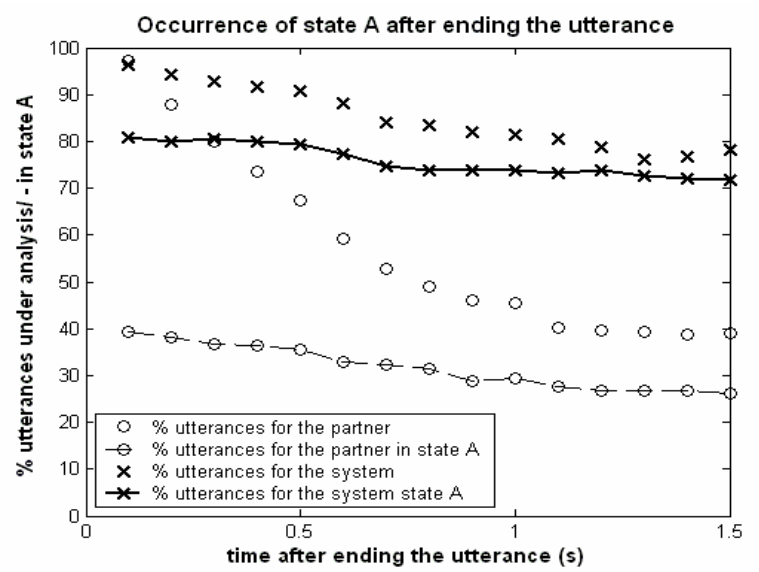

Figure 3: Occurrence of state $A$ after ending the utterance, dots show the percentage of utterances not yet followed up by a new utterance, lines the percentage of utterances still being in state $\mathrm{A}$.

\subsubsection{Classes of the eye gaze feature}

In this section we summarize the chosen features relating to eye gaze. As we have seen we can make a distinction between utterances without transition, looking at the state observed and utterances with transitions, looking at the end state. We consider all other effects too weak to include in the classifier. This leads to eight mutually exclusive categories that cover all utterances:

- No transition, observed state is A,B,C,D

- One or more transitions, end state is A, B, C, D

\subsection{Utterance length}

\subsubsection{Analysis of utterance length}

In [2] we have shown that utterances for the system tend to be consistently short, while utterances for the partner can be both short and long. Therefore utterance length could be used a feature for addressee hood and we examine if this is still the case in this experiment. 
Within this dataset the effect is less salient. With this system [8], users may provide values for multiple search parameters in a single utterance, and in a number of cases people address both the system and the partner within one utterance ("composites"). Still utterance length is a valuable feature as can be seen from Figure 4.

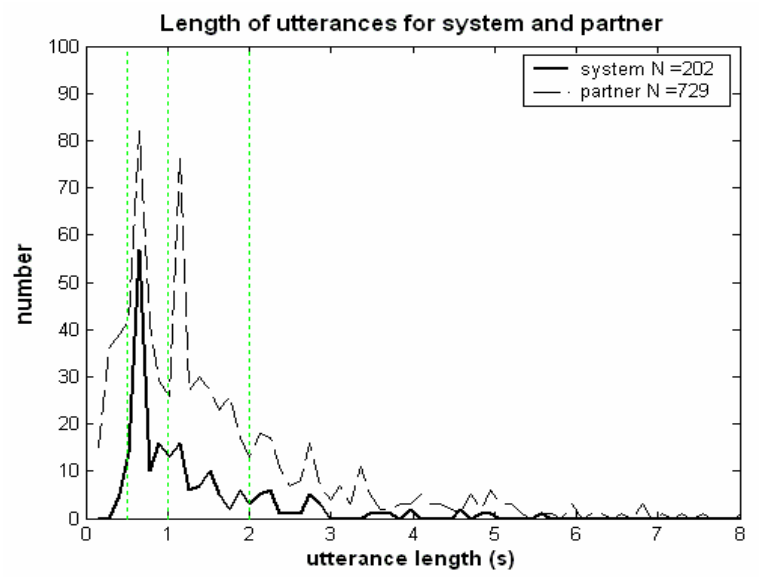

Figure 4: Histogram for utterance length of utterances for the partner (dotted line) and system (plain line)

\subsubsection{Classes of utterance length feature}

A reasonable way of converting the histogram of Figure 4 into a feature is by fitting a probability density function to the histogram with a limited amount of parameters (for instance a single Gaussian, with $\log$ (time) as parameter). However in the classifier of this paper we define 4 categories with a range length. Table 8 shows the chosen ranges and the number of utterances for partner and system in each.

Most utterances for the system are between $0.5 \mathrm{~s}$. and $2 \mathrm{~s}$, utterances for the partner are more evenly distributed. In all classes the number of utterances for the system is lower than the number of utterances for the partner. However this is a result of class skew, and does not play a role in the classifier since we can vary our threshold.

Table 8: Classes used for the utterance length feature

\begin{tabular}{|l|l|l|}
\hline Range & System & Partner \\
\hline $0 \mathrm{~s}-0.5 \mathrm{~s}$ & $19(9 \%)$ & $126(17 \%)$ \\
\hline $0.5 \mathrm{~s}-1 \mathrm{~s}$ & $83(41 \%)$ & $152(21 \%)$ \\
\hline $1 \mathrm{~s}-2 \mathrm{~s}$ & $73(36 \%)$ & $284(39 \%)$ \\
\hline $2 \mathrm{~s}$ or longer & $27(13 \%)$ & $161(22 \%)$ \\
\hline & 58 & 319 \\
\hline
\end{tabular}

\subsection{Dialog events}

\subsubsection{Analysis of dialog events}

In this section we examine whether previous dialog events can serve as a potential feature. As described in section 2 participants had the opportunity to press a button, if they wanted to address the system explicitly. This button was followed by a short question from the system asking for the field input. We expected users to address the system after such an event. Users employed buttons 48 times, 40 times followed by an utterance for the system and 8 times by an utterance for the partner (see also Table 10). Thus pressing a button to explicitly address the system is an indication but not a guarantee for users to address the system. The 8 utterances for the partner can neither be distinguished by looking behavior nor utterance length: in all cases, either the speaker or both participants look at the screen fulltime and the utterance is between 0.5 and $2 \mathrm{~s}$ long.

A second class of dialog events consist of the questions the system asks at relevant points in the dialog. We expect that participants generally will answer the questions. At some point however a question can be a starting point for discussion among participants; for instance, when the system asks "what is the time you want to travel", the participants often start to discuss a suitable time. In total 80 questions were asked by the system, 40 followed by an utterance for the system and 40 by an utterance for the partner (see also Table 10). These utterances can be separated further by looking at the gaze behavior. Table 9 shows this.

Table 9: Gaze behavior after a question from the system

\begin{tabular}{|l|l|l|}
\hline Addressee & System & Partner \\
\hline No Shift & $35(88 \%)$ & $18(45 \%)$ \\
\hline Shift Away & $5(12 \%)$ & $22(55 \%)$ \\
\hline
\end{tabular}

If people start a conversation with the partner against the natural expectations in the dialog (which is to answer the system question) they mark this explicitly with a shift in head orientation. This happens in about half of the cases about half of the cases, whereas the same signal is hardly observed during utterances for the system. Other system events (text appearing on the form, or using a button to fill a field) had no relation with the addressee of subsequent utterances.

\subsubsection{Classes of the dialog events feature}

The system event feature thus contains the following classes:

Table 10: features relating to dialog events

\begin{tabular}{|l|l|l|}
\hline Class (Intended for the system) & System & Partner \\
\hline Not after a system event & $122(48 \%)$ & $675(93 \%)$ \\
\hline After pressing a button & $40(20 \%)$ & $8(1 \%)$ \\
\hline System question, no shift & $35(17 \%)$ & $18(2 \%)$ \\
\hline System question, shift away & $5(5 \%)$ & $22(3 \%)$ \\
\hline All & 202 & 723 \\
\hline
\end{tabular}

\section{DESCRIPTION OF CLASSIFIER}

For classification we use a Naive Bayes classifier. For each feature, head orientation, utterance length, and dialog events and for each class (for instance: both participants look at the system fulltime) we calculate the probability that an utterance falling in that class is for the system.

Equation 1: Probability that an utterance is for the system given that we observe class $x$ of feature $f$.

$$
P(s \mid x, f)=\frac{N(s \mid x, f)}{N(s \mid x, f)+N(p \mid x, f)}
$$

$\mathrm{N}$ is the number of utterances falling in the observed class $\mathrm{x}$; $\mathrm{x}$ can have the values defined earlier on in the paper (Table 3, Table 6 ,Table 7 ,Table 8,Table 10). We make the assumptions that each 
feature gives an equally reliable estimate about the nature of an utterance, and that the features are fully independent.

Equation 2: Probability an utterance is intended for the system given measurements $f_{1}, x_{1}, f_{2}, x_{2}$ and $f_{3}, x_{3}$

$$
P(s)=\frac{P\left(s \mid x_{1}, f_{1}\right)+P\left(s \mid x_{2}, f_{2}\right)+P\left(s \mid x_{3}, f_{3}\right)}{3}
$$

While the dependencies between features are of course of our interest, closer examination is postponed to later on. The purpose of this classifier is rather to get a first order approximation of the combined power of the features we identified.

We obtain the class conditional probabilities from a training set of six out of eight pairs ( 24 dialogs) and we test classification results on a test set of 2 pairs ( 8 dialogs). The classifier is tested using an $\mathrm{N}$ fold cross validation technique, using all 28 permutations of test and training sets, to get a feeling for the variance of the total classification process.

\section{CLASSIFICATION RESULTS AND DISCUSSION}

In a two category classification problem common measures to evaluate performance are precision, recall and the f-measure. However two complications using these measures arise.

1. We have skewed distributions: many more utterances are for the partner; since precision and recall are not independent of the relative occurrence of these two classes they give a limited prediction of classifier performance for different scenarios.

2. Currently it is unknown what the relative user costs are for the different errors, but it seems likely that a falsely rejected utterance for the system is perceived as more disturbing for the interaction than a falsely accepted utterance for the partner.

Therefore we use ROC curves for evaluation (but for convenience we will present Precision and Recall as well). In a ROC curve on the vertical axis the hit-rates are shown:

Equation 3: Hit rate

$$
\text { Hit }- \text { rate }=\frac{N\left(i_{s} c_{s}\right)}{N\left(i_{s} c_{s}\right)+N\left(i_{s} c_{p}\right)}
$$

where $i$ equals the intended addressee and $c$ equals the prediction of the classifier. The hits are the utterances intended for the system divided by all utterances for the system. On the horizontal axis we find the false alarm rate:

Equation 4: False alarm rate

$$
F A-\text { rate }=\frac{N\left(i_{p} c_{s}\right)}{N\left(i_{p} c_{s}\right)+N\left(i_{p} c_{p}\right)}
$$

False alarms are the utterances intended for the partner classified as utterances for the system divided by all utterances for the partner. We can generate ROC curves from our classifier by adjusting the decision bias: adding a weight to respectively the probability of an utterance being intended for the system and the probability an utterance being intended for the partner.
If we want to simplify the performance into a single variable we can take the area under the curve (AUC). In this case we do not address point 2 mentioned above. The AUC for a random classifier is 0.5 .

In Figure 5 the ROC graphs for the features are shown. The solid line indicates the average performance over all test and training sets, the dotted lines the variance ( 2 standard deviations) and the dashed line indicates chance performance.
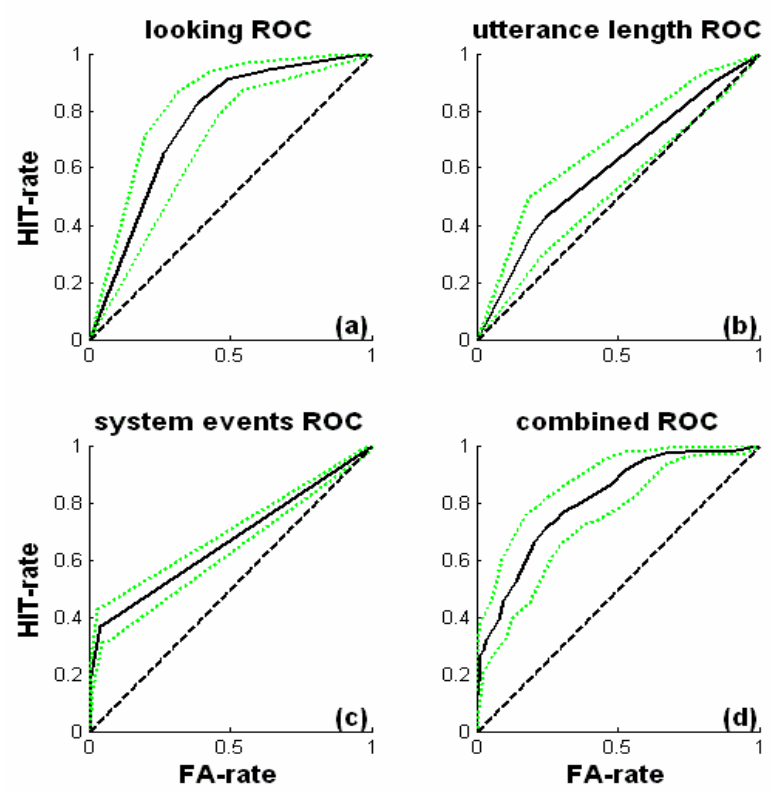

Figure 5: Roc curves for independent features $(a, b, c)$ and their combined performance (d).

Looking behavior turns out to be the strongest feature but it has difficulties avoiding False accepts. Utterance length is the weakest feature having difficulties both avoiding false accepts and producing hits. The system events feature has a high discriminating value, producing hits easily, but operates on only a small set of utterances. This complements looking behaviour very well. The combined performance is reasonably symmetric, and can be tailored towards good hit performance as well as good alarm performance (Precision and Recall for the combination of features are 0.80 and 0.33 , respectively, at optimal accuracy of 0.84). Combining good hit and alarm performance is still very difficult and it is likely that improvements are needed for applying the classifier in a real situation.

A last question to address is the added value of each feature for the final classification. Table 5 lists the independent quality of each feature and the quality of the final classifier without this particular feature. For each AUC the variance (2 standard deviations) is given.

In fact all features can be excluded within the error margin, this is most clear for the utterance length feature: it has the lowest added value for the final classification results. The combined result has smaller variance; as we expected, it is more robust against variation between the behaviors of the different pairs. 
Table 11: Independent AUC's and added value for each feature

\begin{tabular}{|l|l|l|}
\hline Feature & Independent & All but-this feature \\
\hline Looking behavior & $0.75(+-0,14)$ & $0.76(+-004)$ \\
\hline Utterance Length & $0.60(+-0.06)$ & $0.80(+-0.08)$ \\
\hline Dialog events & $0.66(+-0.11)$ & $0.73(+-0.11)$ \\
\hline All & $0.81(+-0.07)$ & \\
\hline
\end{tabular}

The 'addressee is where the eye is' classifier as defined in our earlier paper [2], ("only accept utterances when the speaker looks at the system fulltime") has an AUC of $0.68+-0.18$ on this dataset. This is clearly inferior in both performance and variance.

\section{CONCLUSION AND FUTURE WORK}

We have shown that Eye-gaze, utterance length and dialog events can bet used as features for deciding about addresseehood. We have shown that in realistic scenarios speech centric multi-modal interfaces cannot simply be equated with a third human party in multiparty conversation. This inequality gives us both opportunities and risks in identifying the addressee of utterances.

On the positive side, low level language features such as utterance length can be used because people behave differently towards the system than to the partner. However as the communicative capabilities of these systems improve and users' expectations rise, this difference is likely to diminish.

On the problematic side is the notion of situational attractors; if the visual output of the system supports the task at hand speakers can be expected to look at the system during a large amount of utterances. As a consequence of the a-symmetric gaze behavior our classifier gives acceptable Precision (0.80) but low Recall (0.33). In a human human robot scenario [3], the system did not generate visual output; yet an a-symmetry in gaze behaviour occurred, be it to a lesser extent. We might thus expect to find a similar a-symmetry for a wider range of systems, but most strongly in those cases where the system provides visual support to the dialog.

We may possibly improve classification further using the continuous head orientation data that were also collected during the dialogues; with these data we might be able to distinguish looking away from the partner (toward the system) and looking at the system. Moreover there might be fine-grained patterns in space (rather than time) that we overlook when using discrete data. First explorations of this are promising. We will also explore more sophisticated parametric classification methods than the Bayes classifier presented here.

Further improvements in classification need to be obtained by combining a wider range of features. Besides other non verbal features such as prosodic pitch and loudness, higher level language properties such as used in [3], are candidates. But as their best classification results ( 0.65 precision and 0.81 recall) show: the addition of relating to the language model does not straightforwardly lead to correct classification either. Combining features may help coping with the large individual variances in behavior (see for instance [4]), and in our data set combining features lowered the variance in performance in over de different pairs. Surely (non) verbal communication has a somewhat redundant character. The question of what is a suitable set of features and how they can be best combined is still an open question.

Another route is to improve the actual (gaze) behaviour of the participants. We will explore the effects of giving feedback to the user about the inferences the classifier makes. Well designed feedback design might influence the behaviour of the participants as well as lower the user costs for different types of errors.

\section{AKNOWLEDGEMENTS}

The current research is part of the CRIMI project (Creating Robustness in Multimodal Interaction) (http://crimi.id.tue.nl), and is funded by the Dutch Ministry of Economic Affairs through the Innovation Oriented Programme Man-Machine Interaction (IOPMMI). During the research all authors were affiliated with Eindhoven University of Technology, Ilse Bakx is currently working at the Centre for Usability Research (CUO), Katholieke Universiteit Leuven.

\section{REFERENCES}

[1] Argyle, M. \& Graham, J. (1977) The Central Europe Experiment - looking at persons and looking at things. Journal of Environmental Psychology and Nonverbal Behaviour, 1, 1977, pp. 6-16.

[2] Bakx I., van Turnhout K.G.., \& Terken, J. Facial orientation during multi-party interaction with information kiosks. Proc. of Interact, Zurich, Switzerland, 2003.

[3] Katzenmaier, M., Stiefelhagen, R., Schultz, T. Identifying the Addressee in HumanHumanRobot Interactions based on Head Pose and Speech. Proeedings of. ICMI, State College, PA, USA, 2004

[4] Kendon, A. Some functions of gaze direction in social interaction. ACTA PSYCHOLOGICA, 26, 1967, 22-63

[5] Lang, S., Kleinehagenbrock, M., Hohenner, S., Fritsch, J., Fink, G. A., \& Sagerer G. Providing the basis for humanrobot-interaction: A multi-modal attention system for a mobile robot. Proceedings of ICMI, Vancouver, Canada, 2003.

[6] Rutter, D.R. Looking and Seeing: The Role of Visual Communication in Social Interaction. John Wiley \& Sins Ltd. 1984

[7] Siracusa, M., Morency, L.P., Wilson, K., John W. Fisher III, J.W., Darrell, T. A multi-modal approach for determining speaker location and focus. Proceedings of ICMI, Vancouver, Canada, 2003

[8] Sturm J., Bakx, I., Cranen, B. \& Terken, J. (2002) The effect of prolonged use on multimodal interaction. Proceedings of the ISCA Workshop on Multi-modal Dialogue in Mobile Environments, Kloster Irsee, Germany

[9] Takemae, Y., Kazuhiro O., \& Mukawa N.. Video cut editing rule based on participants' gaze in multiparty conversation. Proeedings of ACM Int. Conf. on Multimedia, 2003

[10] Vertegaal, R., Slagter, R., Van der Veer, G.C. \& Nijholt, A. Eye Gaze Patterns in Conversations: There is More to Conversational Agents Than Meets the Eyes. Proceedings of ACM CHI 2001. Seattle: ACM. 